\title{
La génétique et les rayonnements ionisants
}

\author{
$2^{\mathrm{e}}$ partie
}

\section{Les effets génétiques des rayonnements ionisants}

\author{
B. DUTRILLAUX (*)
}

(Manuscrit reçu le 8 mai 1980)

\begin{abstract}
RÉSUMÉ
Les rayonnements ionisants représentent la catégorie d'agents mutagènes la mieux connue. Il reste impossible de déterminer leur importance relative par rapport aux autres mutagènes possibles. Expérimentalement, on voit que les cellules germinales mâles sont plus sensibles que les cellules germinales femelles. Cette sensibilité est déterminée par la phase cellulaire au moment de l'agression. L'irradiation $\mathrm{X}$ aiguë à forte dose induit un taux de mutation voisin de $1,7.10^{-7} \mathrm{rad}^{-1}$ par gamète et par gène chez le mâle. Ce taux est plus faible en cas d'irradiation chronique. Le caractère pathologique se manifestera en première génération (gènes dominants et anomalies chromosomiques déséquilibrées) ou en $n$-ième (gènes récessifs et remaniements chromosomiques équilibrés).

L'étude directe sur l'homme n'a apporté que des résultats contradictoires, en raison de la difficulté des études épidémiologiques et du faible effet recherché. Parmi les différents émetteurs de rayonnements, seuls les émetteurs $\mathrm{X}$ ou $\gamma$ induisent un véritable risque génétique, les autres rayonnements étant trop peu pénétrants pour atteindre les gonades. La dose de doublement du taux de mutations est estimée à plus de $100 \mathrm{rad}$ chez l'homme et à plus de $1000 \mathrm{rad}$ chez la femme. On ne peut toutefois conclure à l'innocuité de faibles doses, dont l'effet est simplement très difficile à mettre en évidence. Il faut considérer à part le risque individuel, qui reste faible, du risque collectif où le maintien de la qualité du matériel génétique de notre espèce doit demeurer un objectif de première importance.
\end{abstract}

\begin{abstract}
Ionizing radiations are the best known mutagenic agents. Their relative importance as compared to other mutagens cannot be determined. Experiments show that male germinal cells are more sensitive than female germinal cells. This sensitivity is determined by the cell phase at the time of agression. Acute X-exposure results in a mutation rate of about $1.7 \times 10^{-7} \mathrm{rad}^{-1}$ per gamete and per gene in the male. This rate is lower in case of chronic exposure. Pathological effects willappear in the first (dominant genes, and unbalanced chromosomal anomalies) or $n$-th generation (recessive genes and balanced chromosomal rearrangements).
\end{abstract}

(*) Institut de progénèse, 15, rue de l’École-de-Médecine, 75006 Paris. 
Direct studies on humans have brought contradictory results on account of the difficulty of conducting epidemiologic investigations and of the weak effect investigated. Only $\mathrm{X}$ or $\gamma$-emitters induce a true genetic risk, the other radiations being too little penetrating to reach the gonads. The doubling dose of the mutation rate is estimated at over $100 \mathrm{rad}$ in males and over 1,000 rad in females. However, one cannot conclude that low doses are not harmless because their effects are difficult to demonstrate. The individual risk, that remains low, must be distinguished from the collective risk for which the safeguard of the quality of the genetic material of our species must remain our prime purpose.

\section{LES AGENTS MUTAGÈNES}

Nous venons de développer quelles pouvaient être les conséquences des modifications du matériel génétique. Ces modifications qui n'ont d'importance que parce qu'elles sont transmissibles de cellule à cellule, et de génération à génération, peuvent survenir à tout moment. Actuellement, on ignore si une fraction des mutations est strictement spontanée, ce qui signifierait qu'elles pourraient se produire en dehors de toute agression, simplement parce que les systèmes de reproduction cellulaire ne sont pas absolument parfaits. Il serait du plus haut intérêt d'éclaircir ce point, car cela permettrait de savoir si la quasi-totalité de la pathologie héréditaire est induite par notre environnement, ou si elle est simplement le lot de tout système vivant. Ceci est très important pour rechercher le seuil d'action éventuel des agents susceptibles d'intervenir.

Ce qui est beaucoup mieux établi, c'est que certains agents physiques ou chimiques sont susceptibles d'élever la fréquence des anomalies. Parmi les agents chimiques, les analogues des bases entrant dans la composition de l'ADN peuvent jouer un grand rôle. Beaucoup d'autres substances peuvent intervenir à d'autres niveaux, par exemple en atteignant les systèmes de réparation de l'ADN.

Parmi les agents physiques, les seuls dont les effets génétiques aient été clairement démontrés sont les rayonnements ionisants. Les travaux sur ce sujet sont innombrables, et une relation évidente entre la dose reçue et l'effet a pu être mise en évidence. Les rayons ultraviolets sont, eux aussi, capables d'induire des mutations à l'échelle cellulaire, mais ils ne peuvent atteindre les gonades. Chez l'homme, ils n'ont donc aucun effet génétique.

Pour cette raison essentielle, les rayonnements ionisants sont souvent considérés comme le principal agent mutagène. Une telle notion paraîtra probablement périmée dans les prochaines années. En effet, on tend à penser aujourd'hui que le rôle tenu par les mutagènes chimiques est beaucoup plus important que primitivement estimé.

En tout état de cause, les rayonnements sont présentement les facteurs mutagènes les mieux étudiés. Les moyens d'investigation de leurs effets sont multiples, et reflètent directement ce que nous avons vu précédemment : on peut soit apprécier le dégât génique par l'étude de la descendance, en recher- 
chant des caractères anormaux, soit apprécier le dégât chromosomique par l'étude cytogénétique (étude du chromosome). Cette appréciation peut s'effectuer sur la descendance des cellules somatiques, en culture de tissu par exemple, ou encore par l'examen des enfants.

Dans notre espèce, l'expérimentation cellulaire a été poussée, surtout en ce qui concerne l'étude des anomalies chromosomiques où une relation dose-effet est largement démontrée. L'expérimentation sur les cellules germinales étant bien sûr impossible, les études " expérimentales " sont limitées à quelques cas d'irradiation. Finalement, les données admises par notre espèce résultent essentiellement d'extrapolations à partir des résultats obtenus chez l'animal.

\section{RÉSULTATS EXPÉRIMENTAUX SUR L'EFFET GÉNÉTIQUE DES RAYONNEMENTS IONISANTS}

Des expériences consistant à irradier des souris par des rayons $\mathrm{X}$ à raison de 200 rad par génération, et ceci durant plusieurs dizaines de générations sont poursuivies aux États-Unis. L'effet éventuel de cette irradiation est jugé sur la survie, la croissance et la fertilité. Après 45 générations, par exemple, seule une légère baisse de la fertilité a été constatée. Après 200 générations, cet effet n'est même plus observé. De telles études pourraient amener à conclure en l'innocuité quasi totale de telles doses d'irradiation. Toutefois, il faut se garder de conclure ainsi définitivement, car lorsque l'on aborde l'effet sur notre espèce, on se doit d'être beaucoup plus exigeant sur la " qualité » des individus. En particulier, l'atteinte de l'intelligence, qui est un des effets les plus redoutés, passe totalement ignorée dans des études sur la souris. C'est pourquoi l'on préfère maintenant analyser l'effet de l'irradiation sur quelques gènes et extrapoler ensuite à tout l'individu les résultats obtenus, après avoir tenté de préciser le nombre total des gènes qu'il porte.

\subsection{LeS MUTATIONS DE GÈNES RÉCESSIFS}

En fonction de ce que nous avons vu plus haut, ces mutations sont particulièrement difficiles à déceler car l'anomalie n'est détectable ni au niveau chromosomique, ni au niveau de la molécule d'ADN. D'autre part, elles ne se manifestent que si le chromosome paternel et le chromosome maternel sont porteurs du même gène anormal (chez l'homozygote). La seule mutation d'un gène n'induira donc aucun effet visible directement, et il faudra le plus souvent attendre de nombreuses générations pour qu'au hasard des ségrégations, un individu reçoive le même gène anormal de son père et de sa mère.

Quelques études ont été faites chez la souris en analysant, par exemple, la mutation de gènes létaux (qui induisent la mort) chez l'homozygote. Ceci a amené à établir des souches de souris consanguines, où mâles et femelles pouvaient éventuellement porter la même mutation, en un exemplaire, et à juger de la baisse de leur taux de reproduction, par mutation du gène homologue. 
D'une façon générale, ceci a montré que : la sensibilité des cellules germinales mâles est plus grande que celle des cellules germinales femelles; dans les deux sexes, il existe des stades où les cellules sexuelles sont plus ou moins sensibles; la dose totale reçue, le fractionnement et le débit de dose sont des facteurs importants, qui influencent directement les résultats.

Chez les mâles, l'irradiation $\mathrm{X}$ aiguë, à forte dose, des spermatogonies (cellules immatures sensibles) induit un taux de mutations voisin de $1,7 \cdot 10^{-7} \mathrm{rad}^{-1}$ par gamète et par gène.

Ces estimations sont essentiellement basées sur des études effectuées chez la souris : des jeunes mâles ont été irradiés, et l'on a recherché dans leur descendance les mutants pour des caractères pathologiques connus pour être déterminés par des gènes récessifs. Pour faciliter l'analyse des mutations récessives, on peut, par exemple, irradier des hétérozygotes pour un gène donné et apprécier la fréquence des homozygotes dans leur descendance de première génération. Il n'existe aucune donnée précise applicable à la femelle, car les conditions expérimentales sont beaucoup plus difficiles. Il est, toutefois, certain que le taux de mutation est beaucoup plus faible que chez le mâle.

D'autre part, pour extrapoler à l'homme, en tenant compte que, dans les cas " habituels " d'irradiation, la dose et le débit de dose sont notablement plus faibles que dans ces expériences, il faut diminuer d'un facteur 3 environ le taux de mutations, soit $0,6 \cdot 10^{-7} \mathrm{rad}^{-1}$ par gamète et par gène.

$\mathrm{Si}$, maintenant, nous considérons qu'il existe un millier de gènes récessifs susceptibles de muter et de donner un caractère pathologique, nous arrivons à un taux de mutation global, pour l'homme, d'environ $0,6.10^{-4} \mathrm{rad}^{-1}$ par gamète. Rappelons ici nos évaluations du chapitre précédent : du fait de l'accumulation progressive des gènes mutés, on estime qu'en moyenne, chaque cellule sexuelle d'une population normale est porteuse d'une mutation.

\subsection{LeS MUtATIONS DE GÈNES DOMinants}

Ces mutations sont beaucoup plus faciles à mettre en évidence, puisque nous avons vu qu'elles peuvent se manifester d'emblée, lorsqu'un seul des deux chromosomes est porteur d'un gène anormal. Ici encore, les données expérimentales sont fournies par l'expérimentation chez la souris, où les mutations induisant des anomalies osseuses, ou des anomalies oculaires (cataracte), ont été recherchées. Le taux de mutations par gène est estimé voisin de $4.10^{-9} \mathrm{rad}^{-1}$ par gamète (irradiation $\mathrm{X}$ ou $\gamma$ à faible débit de dose).

En supposant, là encore, qu'il existe dans notre espèce environ 1000 gènes dominants susceptibles d'intervenir en pathologie, on obtient un taux de mutation global de $4 \cdot 10^{-6} \mathrm{rad}^{-1}$ par gamète. Comme pour les gènes récessifs, ce taux de mutations de gènes dominants est beaucoup plus faible pour les cellules sexuelles femelles.

\subsection{L'INDUCTION DE REMANIEMENTS DE LA STRUCTURE CHROMOSOMIQUE}

L'anomalie structurale la plus fréquemment induite, parmi celles qui sont transmissibles est la translocation réciproque. Il s'agit d'un échange 
de segments entre deux chromosomes ( fig. $4\left({ }^{*}\right)$ ). Une simple analyse au microscope peut la mettre en évidence. Une autre translocation consiste en la fusion de deux chromosomes. Cette anomalie, bien qu'exceptionnellement radio-induite, est celle qui est le plus souvent observée dans les populations humaines.

Le taux de mutation des remaniements chromosomiques a été estimé chez divers organismes, et il n'est pas exclu qu'il varie d'une espèce à l'autre. Après irradiation testiculaire, on admet qu'il pourrait être de l'ordre de 2 à $9.10^{-4} \mathrm{rad}^{-1}$ par gamète (rayons $\mathrm{X}$ ou $\gamma$, faibles débits de dose). L'irradiation d'ovaires entraîne un taux de mutations chromosomiques beaucoup plus faible.

\subsection{L'INDUCTION D'AUTRES ANOMALIES CHROMOSOMIQUES}

Le danger théorique le plus grave est celui de l'induction de malségrégations qui entraînent la survenue, dès la première génération, d'enfants trisomiques ou monosomiques ( fig. $2(*)$ ). Les nombreuses controverses qui persistent sur cette question montrent que le problème n'est pas entièrement résolu. Toutefois, il est déjà certain que si la fréquence des malségrégations augmente, ce n'est que très faiblement, puisqu'on ne peut rien mettre en évidence dans les conditions habituelles.

\section{CONSÉQUENCES PATHOLOGIQUES PRÉVISIBLES SUR LA DESCENDANCE DES SUJETS IRRADIÉS}

Ce que nous avons développé plus haut montre que toutes les mutations n'ont pas la même importance en pathologie. A l'inverse des mutations de gènes dominants, les mutations de gènes récessifs n'auront, en règle générale, aucune incidence en première génération. Quant aux anomalies chromosomiques, une partie se manifestera en première génération (malségrégations) tandis que les autres n'auront éventuellement d'effet pathologique qu'en deuxième ou $n$-ième génération (translocations).

Une deuxième raison d'hétérogénéité de l'effet pathologique vient de la gravité variable des anomalies génétiques. Les plus graves ne permettent pas le développement embryonnaire ou fætal. L'anomalie entraînera donc une fausse couche, qui sera bien souvent perçue comme une anomalie de la mère et non de l'enfant qu'elle portait. Viennent, ensuite, de nombreuses maladies qui permettent la survie intra-utérine, mais pas ou peu la survie après l'accouchement. De nombreuses anomalies chromosomiques entrent dans ces deux catégories.

Parmi les autres anomalies, celles qui touchent l'intelligence sont des plus redoutées. Les autres affections sont polymorphes et peuvent affecter théoriquement toute fonction. Les anomalies osseuses sont souvent induites par des gènes dominants ou des aberrations chromosomiques, et apparaissent

(*) Voir Première partie.

VOL. $15-\mathrm{N}^{\circ} 4$ 
donc d'emblée. Bon nombre de ces anomalies osseuses ne sont cependant pas préjudiciables, et ne se révèlent souvent qu'à la suite d'un examen radiologique systématique, par exemple.

Il existe donc un paramètre de gravité, dont il faut absolument tenir compte, et ce n'est pas facile, pour déterminer le détriment réel dû aux mutations. Un résumé des risques est fourni par le tableau inspiré des données du Comité scientifique des Nations Unies sur les effets des rayonnements ionisants (U.N.S.C.E.A.R.).

\section{TABLEAU}

EFFet PRÉSUMÉ DE L'EXPosition des POPULATIONS A 1 rad PAR GÉNÉRATION. COMME ON LE VOIT, L'ACCROISSEMENT DE LA FRÉQUENCE DES ANOMALIES, MÊME RÉEL, PASSERAIT TOTALEMENT INAPERÇU, CAR IL SERAIT D'UN ORDRE DE GRANDEUR (inférieur à 2 p. 1000 ) BIEN AU-DESSOUS DU NIVEAU DE PRÉCISION AVEC LEQUEL ON CONNAITT L'INCIDENCE ACTUELLE DES ANOMALIES.

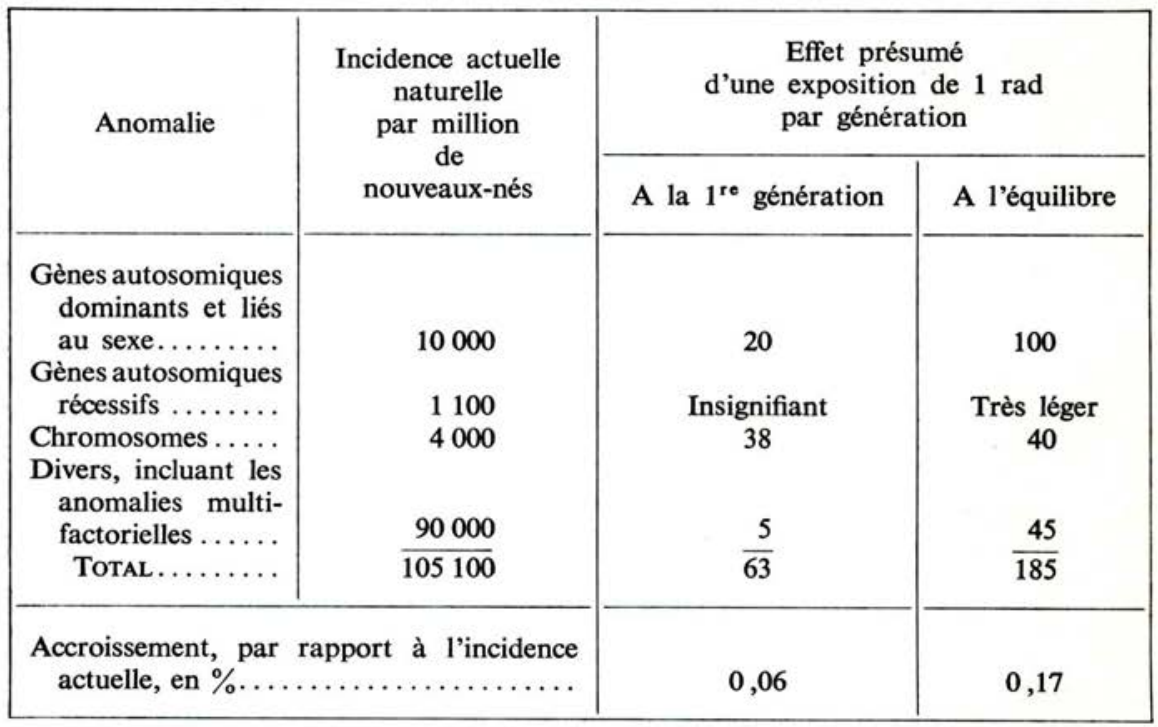

Comme on le voit, ces données, tout à fait théoriques et obtenues en admettant l'hypothèse de la linéarité et de l'absence de seuil de la courbe dose-réponse, sont comparées à la fréquence naturelle des différentes affections. Cette comparaison montre combien il est difficile de juger de l'effet nocif d'un mutagène dans la population humaine.

A la première génération, une irradiation de 1 rad augmenterait la fréquence naturelle des maladies héréditaires d'un peu moins de 1 p. 1000 de sa valeur. A l'équilibre, c'est-à-dire après suffisamment de générations pour que les gènes récessifs et les translocations aient eu la possibilité de s'exprimer, ce qui peut correspondre à plusieurs siècles, l'augmentation serait d'un peu moins de 2 p. 1000. 
$\mathrm{Si}$ nous considérons l'exemple de la trisomie 21 (mongolisme), dont la fréquence naturelle est d'environ 1 p. 750 à la naissance, on voit qu'une augmentation de 2 p. 1000 ferait passer cette fréquence à un peu plus de 1 p. 749. Autrement dit, l'augmentation de fréquence serait si faible qu'elle passerait totalement inaperçue, car nul ne peut dire que la fréquence actuelle est plus proche de 1 p. 745 que de 1 p. 755 . Et pourtant, la trisomie 21 compte parmi les anomalies les moins rares du matériel génétique.

\section{ANALYSE RÉELLE DE LA DESCENDANCE DE SUJETS SOUMIS A DIFFÉRENTS TAUX D'IRRADIATION}

\subsection{Par Radioactivité NatURelle Relativement ÉleVÉE}

L'irradiation naturelle dépend de l'altitude, de la nature des roches et du mode d'habitat. Elle se situe en moyenne autour de $100 \mathrm{mrem} / \mathrm{an}$. D'une ville à l'autre, des variations du simple au double peuvent exister. Des enquêtes réalisées aux États-Unis n'ont révélé aucune variation dans la fréquence des maladies héréditaires en fonction de ce facteur.

Certaines régions de la terre sont particulièrement radioactives. En Inde, (Kerala) et au Brésil, existent des régions où la présence de sables monazites entraîne un taux d'irradiation de 10 à 30 fois supérieur au taux moyen. Malheureusement, les données contradictoires apportées par les études effectuées dans ces provinces ne permettent aucune conclusion, si ce n'est qu'un effet éventuel reste bien difficile à démontrer.

\subsection{PAR IRRADIATION MILITAIRE}

Plusieurs rapports, eux aussi contradictoires, ont été publiés à propos des survivants d'Hiroshima et de Nagasaki et de leur descendance. Une première confusion persiste dans les esprits. Des femmes enceintes irradiées ont fait des avortements ou ont pu donner naissance à des enfants anormaux. Il ne s'agit pas là d'effets génétiques, mais d'effets tératogènes, c'est-à-dire d'une action directe des rayonnements sur les embryons. Parmi les études proprement génétiques, les premiers rapports ont fait état d'un accroissement de la fréquence des anomalies. Ces résultats ont été infirmés ensuite et, actuellement, après étude des deux premières générations de descendants, aucun accroissement significatif des anomalies n'est mis en évidence.

Bien que les doses reçues par les parents aient été assez variables en fonction de leur position par rapport à l'épicentre, on a pu calculer la dose minimale d'irradiation qui serait nécessaire pour doubler la fréquence des mutations. Malgré les imprécisions qui entachent sûrement ces estimations, il semble que la dose doublante serait au moins égale à $130 \mathrm{rad}$ pour les hommes et à $1000 \mathrm{rad}$ pour les femmes. Ceci est de toute manière fort loin de la valeur de $3 \mathrm{rad}$ quelquefois avancée arbitrairement dans certaines publications. 


\subsection{PAR IRRADIATION MÉDiCALE}

Il est bien connu que cette source d'irradiation est la principale touchant les populations médicalisées. Différentes études de la descendance de mères irradiées ont été faites dans divers pays. Là encore, les résultats sont contradictoires, et un accroissement faible de la trisomie 21, par exemple, a été avancé par certains et réfuté par d'autres. L'analyse des résultats est encore compliquée par le fait que l'état de santé qui a conduit à faire subir à ces femmes des examens radiographiques peut aussi être rendu responsable de la naissance d'enfants anormaux. Pour conclure, il apparaît que ces différentes études sont incapables de montrer un accroissement de la fréquence des maladies génétiques consécutif à une irradiation.

Cependant, ces données ne permettent pas de conclure à l'inefficacité génétique de faibles doses de radiations. En effet, s'il existe un dommage génétique mais très faible, il ne pourrait pas être mis statistiquement en évidence par les enquêtes épidémiologiques dont nous disposons actuellement. Il faudrait étudier une énorme population pour pouvoir parvenir à le détecter. Dans l'hypothèse d'une linéarité et d'une dose doublante de l'ordre de 130 rad (admise d'après l'analyse des descendants mâles d'Hiroshima et de Nagasaki), une dose de $30 \mathrm{rad}$ ferait passer la fréquence de la trisomie 21, par exemple, de 1/750 à 1/650 environ, c'est-à-dire qu'elle entraînerait une augmentation réelle, mais que les enquêtes épidémiologiques n'arriveraient pas à révéler avec certitude.

Finalement, on peut seulement conclure qu'en raison de la faible fréquence des maladies héréditaires et des difficultés de l'analyse des populations humaines, il ne faut pas attendre de renseignements précis des enquêtes épidémiologiques. Pour que ces enquêtes soient réellement informatives, il faudrait qu'elles s'appliquent à des populations fortement irradiées pour que l'effet soit sensible, et fortement médicalisées pour que l'effet soit reconnu. Les problèmes génétiques résultant d'une telle irradiation (plus de $100 \mathrm{rad}$ ) seraient alors en arrière-plan par rapport aux risques somatiques.

\section{INFLUENCE DE L'AGE SUR LES RISQUES GÉNÉTIQUES}

La sensibilité des cellules sexuelles et les conséquences de leur irradiation dépendent directement du mode de fabrication des cellules sexuelles et des habitudes de reproduction.

Chez la femme, les cellules sexuelles commencent leur maturation dès l'âge fœtal. Elles restent ensuite bloquées jusqu'à la puberté, et termineront leur maturation à chaque cycle, jusqu'à la ménopause. La période de reproduction potentielle s'étend en moyenne sur 30 ans, de l'âge de 15 ans à celui de 45 ans environ.

Il existe finalement deux périodes particulièrement sensibles. L'une, brève, va jusqu'au $7^{\mathrm{e}}$ mois de la vie fœtale. On voit ainsi que l'irradiation 
de femmes enceintes peut avoir des conséquences génétiques directes pour la descendance de l'enfant qu'elles portent. L'autre période sensible est celle de la reproduction. De la naissance à la puberté, les études expérimentales chez l'animal indiquent une grande résistance des cellules sexuelles à l'irradiation. Enfin, c'est une évidence, l'irradiation de femmes post-ménopausiques n'a aucun effet génétique.

Chez l'homme, les cellules sexuelles ne commencent à entrer en maturation qu'à la puberté. Auparavant, il n'existe que des cellules non matures, les spermatogonies, qui semblent peu sensibles à cette période où elles sont inactives. La fabrication des cellules sexuelles, une fois commencée, est continue et se prolonge jusqu'à la fin de la vie. Théoriquement donc, l'irradiation d'un homme, à tout âge, peut entraîner des conséquences génétiques. Toutefois, au moins dans les pays occidentaux, l'immense majorité des paternités est attribuée à des hommes dont l'âge est compris entre 20 et 40 ans.

Le risque génétique collectif résulte donc, essentiellement, de l'irradiation cumulée par les adultes en âge de se reproduire. Sur le plan individuel, il est bon de savoir qu'en raison de l'existence des processus de réparation des dégâts, le risque génétique s'atténue à mesure que l'on s'éloigne de la période d'irradiation. En pratique, après une forte irradiation accidentelle ou thérapeutique, on conseille d'attendre 6 mois environ avant de chercher à procréer.

\section{INFLUENCE DU TYPE DE RAYONNEMENT ET DES MODALITÉS D’EXPOSITION}

Les rayonnements de différentes natures $(\alpha, \beta, \mathrm{X}, \gamma$, neutrons, ...) peuvent tous induire des mutations bien que les relations entre dose et effet soient très différentes, dans leur forme, selon la valeur du transfert linéique d'énergie (TLE) ou densité d'ionisation des particules chargées, primaires ou secondaires, qui délivrent la dose au tissu. Encore faut-il que ces rayonnements atteignent les cellules germinales (gonades).

Dans le cas de l'irradiation externe, seuls les rayonnements dits " pénétrants ", $\mathrm{X}, \gamma$, neutrons, peuvent atteindre ces cellules qui sont situées grossièrement à $1 \mathrm{~cm}$ de profondeur chez l'homme et à $7 \mathrm{~cm}$ chez la femme. Les rayonnements $\alpha$ ont des parcours de quelques dizaines de micromètres dans les tissus et sont entièrement absorbés par la couche cornée de la peau; quant aux électrons, ce n'est que pour des énergies supérieures à $2 \mathrm{MeV}$ qu'ils peuvent atteindre la profondeur de $1 \mathrm{~cm}$.

Dans le cas de la contamination interne, aucun émetteur $\alpha$ ne se localise préférentiellement dans les gonades et, dans le cas du plutonium par exemple, on estime à 3 et à 1 dix millième respectivement la fraction du plutonium présent dans l'organisme qui se localise dans les gonades de l'homme et de la femme [11]. Certains chercheurs ont même mis en doute, compte tenu de la localisation fine du plutonium dans les tissus des glandes sexuelles mâles, 
la possibilité pour les rayons $\alpha$ émis par le plutonium d'atteindre les cellules germinales chez l'homme. Quoiqu'il en soit, l'effet génétique éventuel d'une contamination par le plutonium peut être considéré comme négligeable.

Beaucoup d'émetteurs $\beta$ et $\beta \gamma$, par contre, se répartissent d'une façon relativement homogène dans les tissus mous (tritium, potassium, césium, ...) et peuvent ainsi entraîner une certaine dose au niveau des cellules germinatives; le risque est alors, à dose égale, analogue à celui que peut entraîner l'irradiation externe par les rayonnements $\mathbf{X}$ et $\gamma$.

\section{CONCLUSIONS}

La pathologie génétique reste quelque peu mystérieuse car elle est constituée de centaines de maladies, toutes d'une grande rareté. Beaucoup de ces maladies sont congénitales et sont, de ce fait, souvent confondues avec d'autres affections, elles aussi apparentes à la naissance, mais sans origine héréditaire. Du fait de cette rareté, la fréquence de chacune des maladies génétiques n'est pas connue avec une grande précision. De la sorte, il est extrêmement difficile, pour ne pas dire impossible, de rechercher un accroissement faible de leur survenue. L'exemple des descendants des irradiés d'Hiroshima et de Nagasaki est, à cet égard, démonstratif puisque les enquêtes ne montrent pas d'effet.

Dans l'état actuel de nos connaissances, il importe donc d'avoir recours à l'expérimentation et, pour des raisons pratiques, la souris est l'animal le plus utilisé. L'essentiel des données appliquées à l'homme résulte donc d'une extrapolation à partir de cet animal, et l'on conçoit que de nombreuses erreurs puissent en résulter.

Conscients de cette difficulté, les experts ont donc toujours effectué leurs extrapolations de façon très pessimiste. Il en résulte, peut-être, une surestimation des risques génétiques, mais la prudence la plus élémentaire veut que nous acceptions les données chiffrées comme si elles avaient une valeur absolue. Il est très vraisemblable que ces données vont s'affiner au cours des prochaines années, car les expériences animales se poursuivent, se précisent, et la pathologie héréditaire est de mieux en mieux connue. Pour l'heure, les estimations fournies par les différents organismes internationaux compétents en la matière (Comité scientifique des Nations Unies, Commission internationale de protection radiologique) ou nationaux, sont toutes basées sur les mêmes expérimentations et sur les mêmes enquêtes statistiques de la population humaine. Les petites variations dans les estimations que l'on peut y déceler ne doivent pas laisser croire en leur mal fondé. Elles reflètent simplement les différentes façons, plus ou moins pessimistes, d'effectuer les extrapolations nécessaires.

Bien que différentes, ces estimations restent toutes dans le même ordre de grandeur. Elles montrent que, pour des doses faibles d'irradiation, l'accroissement du risque génétique est faible. Si petit, que certains pourraient vite conclure à l'innocuité des faibles doses (de l'ordre de quelques dizaines de 
rad), puisqu'on ne décèle rien. Mais rappelons que, du fait de la très faible fréquence naturelle de chaque maladie, on ne peut détecter que les accroissements forts. Nous ne pouvons donc être certains de l'innocuité des faibles doses, dans l'absolu.

Rappelons aussi que l'une des caractéristiques des maladies héréditaires est d'être déterminées par des gènes, ou des remaniements chromosomiques, qui peuvent rester muets pendant des générations, mais qui peuvent aussi s'accumuler. Cette accumulation accroîtra très lentement le fardeau génétique des générations futures, et c'est une question de principe que de chercher à préserver le patrimoine héréditaire de notre espèce. Les recommandations des organismes internationaux de protection ont d'ailleurs toujours considéré cet objectif comme essentiel.

Mais il faut aussi considérer les bénéfices éventuels liés à cette nuisance. Ainsi, par exemple, bien que l'utilisation médicale des rayonnements ionisants à des fins de diagnostic radiologique soit la source principale d'irradiation de la population française, il est hors de doute que nul ne songe à la supprimer; néanmoins, on pourrait accroître les efforts pour réduire l'exposition des patients, ce qui est très réalisable.

Finalement, il reste fort difficile au généticien de proposer une ligne de conduite univoque à notre société vis-à-vis des rayonnements ionisants. Si l'on considère le risque génétique collectif, il faut, par principe, limiter le plus possible toute exposition. Si l'on considère le risque individuel, il faut savoir que l'exposition annuelle à quelques rads ne l'accroît pas d'une manière appréciable. Il faut, dans tous les cas, replacer les nuisances résultant de l'irradiation parmi toutes les autres dont les effets nocifs éventuels sont encore moins bien connus et peut-être supérieurs. On ne peut donc que souhaiter le développement d'études qui nous permettraient de mieux connaître ces autres facteurs.

\section{BIBLIOGRAPHIE}

[1] BEIR III. Report of the Committee on the biological effects of ionizing radiation, Washington, National Academy of Sciences, 1980.

[2] Cavalli-Sforza L. L., Bodmer W. S. Génétique des populations humaines, San Francisco, W. H. Freeman, 1971.

[3] Recommandations de la Commission internationale de protection radiologique. Publication C.I.P.R. 26, Oxford, Pergamon Press, 1979.

[4] Crow J. F. Can we assess genetic risks? In: 6 International Congress of Radiation Research, Tokyo, 13-17 mai 1979 (OKADA S. et al., Ed.), Tokyo, Japanese association for radiation research, 1979, 70-78.

[5] Lamy M. Génétique médicale, $2^{\mathrm{e}}$ éd., Paris, Masson, 1975.

[6] Lamotte M. et L'Heritier Ph. Biologie générale. 2, Lois et mécanisme de l'hérédité, Paris, Doin, 1966.

[7] Surton H. E. An introduction to human genetics, New York, Holt, Reinhart and Winston, 1975.

vol. $15-\mathrm{N}^{\circ} 4$ 
[8] Stern C. Principles of human genetics, 3rd ed., San Francisco, W. H., Freeman 1973.

[9] U.N.S.C.E.A.R. Rapport du Comité scientifique des Nations Unies pour l'étude des effets des rayonnements ionisants, New York, Nations Unies, 1977.

[10] Wallace B. Génétique des populations, adapté de l'anglais par Lucotte G., Paris, Masson, 1974.

[11] Richmond C. R. et Thomas R. L. Plutonium and other actinide elements in gonadal tissue of man and animals, Health Phys., 1975, 29, 241-250. 